\title{
A New Look at Cavitation and the Applications of Its Liquid-Phase Effects in the Processing of Food and Fuel
}

\author{
Muthupandian Ashokkumar (Corresponding author) \\ School of Chemistry, University of Melbourne \\ Parkville, Victoria 3010, Australia \\ Tel: 61-383-447-090_E-mail: masho@unimelb.edu.au \\ Olga Krasulya \& Sergey Shestakov \\ Moscow State University of Technology and Management, Russia \\ E-mail: okrasulya@mail.ru,sdsh@mail.ru
}

Raul Rink

Oil Tech Production OY, Estonia

E-mail: info@oiltech-nordic.eu

$\begin{aligned} & \text { Received: September 21, } 2011 \quad \text { Accepted: October 11, } 2011 \quad \text { Published: February 1, } 2012 \\ & \text { doi:10.5539/apr.v4n1p19 }\end{aligned} \quad$ URL: http://dx.doi.org/10.5539/apr.v4n1p19

\begin{abstract}
Ultrasound-induced acoustic cavitation has been studied in detail due to its chemical effects (sonochemistry) and light emission (sonoluminescence). However, the physical effects such as shear, shockwaves, etc., can also be used for useful applications despite their harmful effects, such as cavitation erosion. It has been suggested that the physical forces generated during cavitation may alter the 3-dimensional network of water molecules and hence a better hydration of macromolecules can be achieved using the cavitation process. In recent times, the physical effects of acoustic cavitation are used in food processing applications. The possibility of using hydrodynamic cavitation for food processing as an alternative approach to ultrasonic processing has been discussed in this manuscript. A special rotary disintegrator, developed by Dr. Hint in the last century is used for the generation of efficient hydrodynamic cavitation. A mathematical model has been developed for the optimization of rotary disintegrators. The suitability of the model for evaluating the process efficiency has also been tested using experimental data obtained for the production of emulsions used as fuels. The development and testing of a new mathematical model for optimizing rotary disintegrators pave new pathways to the use of hydrodynamic cavitation for processing large volumes of liquid ingredients making them suitable for fuel and food industries.
\end{abstract}

Keywords: Acoustic cavitation, Hydrodynamic cavitation, Food processing

\section{Introduction}

Ultrasonics and sonochemistry are relatively new disciplines that are of interest to chemists and physicists due to the fundamental and applied aspects of these interdisciplinary processes. Owing to the unusual chemical reaction environment generated during ultrasound-induced acoustic cavitation, the European Universal Decimal Classification (UDC) classifies sonochemistry to high energy chemistry (UDC 544.57), but the American subject index (PACS) to a section on physical acoustics PACS 43 (Acoustics), PACS 43.35.Vz (Chemical effects of ultrasound). Acoustic cavitation has been used in many applications that include materials synthesis and food processing. One of the issues associated with the use of ultrasonics and sonochemistry in industrial scale applications is the scale-up issues. In this manuscript, hydrodynamic cavitation is proposed as an alternative to acoustic cavitation for large scale processing applications. The authors have attributed this work to UDC 532 (Physics. Hydromechanics) since it deals with hydrodynamic cavitation using a rotary disintegrator and the efficiency of this system is compared with that of acoustic cavitation. 


\section{Acoustic Cavitation}

Acoustic cavitation refers to the ultrasound-induced growth and collapse of existing bubble nuclei in a liquid. The near adiabatic collapse of the bubbles generates extreme temperatures within the collapsing bubbles generating chemical reactions and light emission (sonoluminescence, SL) (Nigmatulin, et al., 2005; Margulis, 1995; Flannigan \& Suslick, 2005). The mechanism of SL emission has been explored in detail. It is generally accepted that SL arises from a combination of plasma and fluorescence, at least in during multibubble cavitation (MBSL) (Margulis, 1995; Flannigan \& Suslick, 2005). In single bubble (SB) cavitation, SBSL is shown to fit blackbody emission under specific conditions (Margulis, 1995; Flannigan \& Suslick, 2005). However, emission due to excited state chemical species has also been observed.

The debate that unfolded during 2002-2007 on attempts to create sonofusion by MB cavitation in deuterated acetone (Taleyarkhan, et al., 2002, 2004; Nigmatulin, 2005; Lahey, et al. 2007; Khavroshkin O., Bystrov, 2007; Knapp, et al., 1970) showed that the knowledge of acoustic cavitation continues to build on uncertain predictions. The mathematical description of the cavitation phenomenon generated by an elastic acoustic wave propagating in liquids is limited to references that deal with the differential equations of motion of the wall of a cavity under the influence of a harmonically varying external pressure (Khavroshkin O., Bystrov, 2007; Knapp, et al., 1970; Shestakov, 2001; Klotz, Hynynen, 2010; Rozenberg, 1968) - one of the solutions proposed by Rayleigh in the last century. A generalized approach to estimate the energy dissipated by cavitation based on empirical data was formulated in the work carried out in Russia under the leadership of Professor Rosenberg (1968). A mathematical description of the spatial distribution of energy transformed by an ensemble of cavitation bubbles, controlled by an external harmonically varying pressure (the field of cavitation), began to emerge only recently (Mettin R., et al., 2006; Shestakov, 2005, 2008, 2010). In the papers published by Shestakov $(2008,2010)$, the problem of modeling the energy of cavitation field is solved by finding the power density distribution of cavitation in the coordinate space of the standing wave in a liquid, which is closed inside rigid boundaries. This is performed based on the known fact that the damping pressure perturbations by the pulsations of a bubble are inversely proportional to the distance, whereas cavitation erosion is inversely proportional to the square of the distance.

Assuming that cavitation is an ergodic process and considering that the pressure pulse from the bubbled, $I_{p}=\int \widetilde{p} \mathrm{~d} t$ is a phase function, the average characteristics of cavitation in the geometric space are replaced by the average values of dimensionless time of the cavitation process. Such an approach is used to describe the total pressure perturbation of cavitation in each period of the harmonic wave. At the same time, the time derivative of the $\delta$-Dirac function is used to describe the total pulse pressure of cavitation, greatly simplifying the mathematical expressions. This model provided an opportunity to describe the effect of the pressure pulse generated by the pulsation of one bubble on the oscillation of other bubbles (Margulis, 2004). On the basis of this model, computer programs were created to construct the distribution functions of the power of cavitation in the field of a harmonic wave in a liquid. To create sonofusion in MB, the authors have suggested a cluster collapse model (Lahey, et al., 2007) - a hypothesis on the possibility of nonparametric amplification of cavitation by the energy generated during the collapse of bubbles in a cluster. The noise of cavitation is not coherent, and hence the superposition of pulses with the same phases in a particular point in space is a random phenomenon. This hypothesis is limited to the introduction a stochastic component in the model. In other words, the model has been used in experiments as a way to control the spatial distribution of the likelihood of the nonparametric amplification of cavitation (Krefting, et al., 2004). However, to increase the cavitation bubble temperature in accordance with the existing theory (temperature in which measured by the law of blackbody radiation), the amplitude of the sound pressure should be increased, as reported in the literature for a SBSL case (Flannigan \& Suslick, 2005). However, for a similar increase in MBSL intensity in aqueous $\mathrm{NaCl}$ solutions an increase the acoustic power was not required. In addition, the deterministic part of this model of cavitation in conjunction with the principles of theory of physical phenomena can perform engineering calculations and predict the characteristics of reactors and optimize the efficiency of specific processes (Dezhkunov, et al., 2007). Thus, problem of building equipment for the application of sonochemical reactions in the industry, based on acoustic cavitation, has been solved from a theoretical point of view. This theory of nonparametric increase of the cavitation in some way resonates with the theory of enhancing the cavitation activity by modulating the ultrasound (Shestakov \& Krasulya, 2010), but the modulation is due to the pressure pulse generated by cavitation bubbles themselves and hence a more complicated model needs to be considered.

Sonochemical reactions in general are considered to be generated by the primary radicals generated within the bubbles and other secondary reactions that occur at the bubble solution interface or in the bulk liquid (Ashokkumar, et al., 2008). However, there are several reactions or applications that depend upon the physical effects generated by cavitation. For example, in food processing applications (Ashokkumar, et al., 2010; Tikhomirova, et al., 2011), reactions induced by the primary radical are not required. One such application that has been recently reported is in 
food processing. More specifically, the sonication of an aqueous dispersion of whey proteins has increased the heat stability of the whey proteins as shown in Figure 1 (Patent claim WO 2007111524 International application PCT/RU2006/000140 / Biopolymer hydrating method, 2006). The heating of whey proteins in an aqueous solutions leads to an increase in solution viscosity due to protein denaturation and aggregation. The sonication of this high viscous solution for less than a minute has reduced the viscosity to that of the original (native) protein solution. Further heating of the sonicated solution does not increase the viscosity as seen in Figure 1. Here, the physical forces generated during acoustic cavitation have changed the surface properties of the protein aggregates leading to an increase in the heat stability. In fact, most of the reactions are independent of the chemistry that occurs within the cavitation bubbles. Some examples include the denaturation of biopolymers, restructuring of the hydration shells around ions, dispersion of sols, etc. (Ashokkumar et al., 2010; Tikhomirova, et al., 2011; Duckworth, 1975).

Tikhomirova et al. (2011) have shown that sonication may lead to the changes in the hydration of ions that are important in food chemistry (Reaction 1).

$$
n \mathrm{H}_{2} \mathrm{O} \rightarrow\left(\mathrm{H}_{2} \mathrm{O}\right)_{n}+Q
$$

where $Q=25-29 \mathrm{~kJ} / \mathrm{mole}$. A proper use of this reaction allows the control of the hydration of biopolymers in food (Mawson \& Knoerzer, 2007). This is a very important process in food industry (Akbarzade et al 2007), because human society is now forced to keep food resources in dried or frozen state, making the hydration process crucial during the addition of water to dried food during the preparation process for consumption. In addition to this, radical reactions generated by acoustic cavitation should be avoided during ultrasonic processing of food ingredients. It has been suggested that the choice of ultrasound frequency is an important parameter in food processing applications (Tikhomirova, et al., 2011). Despite the development of lab-scale food processing applications, scale-up of the processing to industrial applications is an issue (Shlenskaya, et al., 2011). One of the approaches to the problem of scale-up of acoustic cavitation is to find an alternative process that can cause similar effects of cavitation. Hydrodynamic cavitation seems to generate strong physical effects as that of acoustic cavitation but to a greater extent (Shlenskaya, et al., 2011; Hint, 1981; Promtov, 2001). Due to the shortcomings of ultrasonics processing, a new look at the hydrodynamic method has emerged for producing cavitation and for the possibility of its use in food processing.

\section{Hydrodynamic Cavitation}

Professor Knapp at the University of California has mentioned in his book on hydrodynamic cavitation that "an unpleasant hydrodynamic phenomenon, the harmful effects of which often creates serious difficulties in solving many scientific and engineering problems". The quote refers to the problems faced by hydromechanics due to hydrodynamic cavitation owing to the destructive forces generated during this process. In applied research, to prevent the harm or, conversely, to search for useful applications of effects generated by hydrodynamic cavitation, mainly of cavitation erosion, the process needs to be quantified. This can be achieved by measuring the acoustic noise generated by cavitation. In the areas of sonochemistry and hydrodynamic cavitation, the erosion effect, which in the area of fluid mechanics is a cause of destruction, is considered as a positive factor. Here, we describe hydrodynamic cavitation disintegrators (Fig. 2), which are based on the hypothesis of Dr J. Hint (Estonia). It can be expressed by the following quote from his essay: «... the more the number of strokes imparted by particulate matter, the greater the impact velocity and the smaller the interval between successive blows, the more there is activity» (Promtov, et al., 2001). From this assessment, similar representations of disintegrators are set forth in the development of rotary-pulse devices, where the efficiency of hydrodynamic cavitation is increased by increasing the rate of fluid flow and interruptions to the flow rate.

\subsection{Theory}

The main factor in hydrodynamic cavitation is the kinetic energy of the fluid flow, which is proportional to the square its velocity (Promtov \& Monastirsky, 2000; Balabyshko, 1988). It is easy to realize in such rotor-switching device that processing $10 \mathrm{t} / \mathrm{h}$ of fluids, at a speed of $300 \mathrm{rps}$, can be achieved using a rotor diameter of $150 \mathrm{~mm}$ and a $10 \times 20 \mathrm{~mm}$ channel. The flow of the processing liquid occurs as long as these channels have openings with little more than a tenth of a millimeter. When gas bubbles move from a high to a low pressure zone, they do not survive. The sudden drop in pressure leads to the growth and the eventual collapse of these gas bubbles, referred to as hydrodynamic cavitation. In rotary disintegrators, the effects are not just due to the liquid flow alone - additional forces are generated due to hydrodynamic cavitation. Here, we should use a slightly different approach to assessing the efficiency than commonly used. The pulsations of cavitation bubbles and the shock waves that emanate from them can be major forces causing cavitation erosion, which in the theory of vibrations and waves can be estimated by finding the value of the deformation of rarefaction and compression, that is, determining the magnitude of the 
potential component of the scattered energy in a fluid. Cavitation power is proportional to the square of the acoustic pressure. It depends not so much on the changes in pressure caused by the discontinuity of fluid flow through channels in the radial direction, but it is generated by movement relative to the profile of transversal section of the rotating rotor, which is due to the presence of periodically repeating holes.

The latter can be confirmed by looking at the homogenizers manufactured by Aquametro AG, in which the fluid does not flow in the radial direction but axially in the gap between deaf equipped with grooves facing each other on the walls of the rotor while the stator cross-sectional area remains constant (Figure 3a). Among the rotary disintegrators widely used, for example, where the rotor and stator are used with one or more cylindrical shells of finite length with bottoms, fluids flow through the hole of rectangular shape. The rotor and stator create a working volume between the top and the bottom of one another, fluid flows through the axis to the periphery of the construction in the radial directions by opening and closing of the rotating liquid flow channels (Figure $3 b$ ). Mathematical model of cavitation in a disintegrator may be based on differential equations of Hickling-Plesset or Rayleigh-Plesset for the motion of bubble wall under the influence of strain and stress, as described in some problems of solid mechanics by theory of functions of complex variables. The mechanical stress in the fluid, that is, the pressure at any it point, can be estimated on the basis of conformal mapping. For example, in order to describe the pressure distribution in the region $z$ in the liquid (Figure 3c) through the region, which has a uniform distribution of stress and strains such as an infinite strip of constant width $\zeta$ (Figure 3d), can be used the Schwarz-Christoffel integrals.

In such a model, the liquid does not have the friction on the structural elements of the cage, but the rheological equation should have the friction term as in an absolutely elastic body. To calculate the absolute values of the characteristics, these conditions are not quite correct. However, in a comparison of similar operations on the same liquid, they are acceptable, especially for the cavitation bubbles that behave as a Newtonian fluid. Mechanical stress at any point can be expressed in terms of the pressure in the working volume $p_{0}$ and derivative of the mapping function to the invariant $\zeta$ :

$$
\sigma=\frac{p_{0}}{\dot{\mathrm{z}}^{2}}, \text { where } \dot{\mathrm{z}}=\frac{\mathrm{dz}}{\mathrm{d} \zeta}
$$

It is clear that the exponent at $\dot{z}$ is 2 only if all the profiles of fluid flow plane-parallel to each other. The nature of the elastic strain and stress at any point of the fluid, as well as their behavior under the action of cavitation in liquids can be determined by numerical simulation. They allow for numerical comparisons. The behavior of the cavitation bubble can be modeled, for example, by numerical integration of Hickling-Plesset equation as performed by Runge-Kutt. The periodic changes of the pressure in fluid relies upon (Equation (2)) the derivative from the conformal mapping of infinite strip in diametrical cross-section of one hole in the rotor, one hole in the stator and the gap between them:

$$
\mathrm{z}=\frac{\delta}{\pi} \operatorname{Arth} \frac{\operatorname{sh} \frac{\pi}{2} \zeta}{\sqrt{\operatorname{ch}^{2} \frac{\pi}{2} \zeta+\frac{a^{2}}{\delta^{2}}}}+\frac{a}{\pi} \operatorname{arctg} \frac{a \operatorname{sh} \frac{\pi}{2} \zeta}{\sqrt{\delta^{2} \operatorname{ch}^{2} \frac{\pi}{2} \zeta+a^{2}}}
$$

Where, $a$ - the size of the hole on arc of diameter (width of the hole); $\delta$ - size of the gap between the stator and rotor; $\zeta$-coordinate on the invariant expressed by the complex number $\xi+j \eta$. Its derivative by $\zeta$ :

$$
\dot{\mathrm{z}}=\sqrt{1+\frac{a^{2}}{\delta^{2}\left(2+e^{\pi \zeta}+e^{-\pi \zeta}\right)}}
$$

Reciprocal of the square of $\dot{\mathrm{z}}$ is proportional to the tensile deformation of the fluid at any point, causing a pressure change in it.

To calculate the pressure change in the points on the real axis for the total period, the coordinate of the invariant must vary in the range $\pm \xi$. The time during which a corresponding change in pressure occurs will be, $T=\frac{\xi}{\omega \pi R}$ , where $R$ is the outer radius of the rotor, and corresponds the minimum allowable period changes of pressure on 
the elastic deformation of the liquid. $\xi$ is a root of the transcendental equation:

$$
\varepsilon=1-\frac{\delta^{2}\left(2+e^{\pi \xi}+e^{-\pi \xi}\right)}{\delta^{2}\left(2+e^{\pi \xi}+e^{-\pi \xi}\right)+a^{2}},
$$

Where, $\varepsilon=0,05-$ margin of error in relative units.

Quantification of the effectiveness of cavitation can be performed on the increment of the extent of erosion using conditional index, $\Delta P$ (unit capacity of erosion). That is, the full capacity can be estimated during the full compression on the wall of one of the bubbles (when $p_{\max }>p_{0}$ ), located in each channel in the middle of the gap between stator and rotor at maximum for the period its volume $V_{\max }$ :

$$
\Delta P=\frac{\beta}{2} \omega V_{\max } N n\left(p_{\max }-p_{0}\right)^{2},
$$

where: $\beta$-adiabatic compressibility of the fluid; $\omega$ - rotor speed; $N$ - number of channels in the rotor, $n$-in the stator. The model was implemented in a computer program.

\subsection{Calculations and Experiments}

The model was tested by performing a large number of computational experiments over a wide range of variation of parameters of rotary disintegrator. For this, water was chosen as the liquid. Based on the information presented in the literature (Floris, 2004; Promtov \& Monastirsky, 2000), the behavior of bubbles with a diameter $10 \mu m$ was evaluated.

Next, a comparison was made using the disintegrator available in "Oil Tech Production" company (Fig. 2) and rotary-pulsating disperser РИКА-200, by mathematical modeling in accordance with (2) - (7).

The results of this comparison are shown in Table 1, where for the parameters $N, n$ and $\Delta P$ through fraction indicates the values at the boundaries the range of parameter $a$.

Results of the computation experiments with $\omega=100 \mathrm{~s}^{-1}, \delta=100 \mu \mathrm{m}$, and $R=0,1 \mathrm{~m}$ are shown in Figs. 4 and 5. The full-scale experiment with disintegrator of «Oil Tech Production» company was conducted with representatives from the Experimental Laboratory of Fuels of the Tallinn Technical University with oleophilic emulsions containing $20 \%$ aqueous phase. The level of the cavitation erosion in the aqueous phase in $d B$ was determined using (8):

$$
P_{\text {cav }}=10 \cdot \lg \left(10^{0,1 P_{\text {em }}}-10^{0,1 P_{\text {oil }}}\right),
$$

where, $P_{\mathrm{em}}, P_{\mathrm{oil}}$-are the noise levels at a frequency of $8,7 \mathrm{kHz}$, measured in $d B$, in the processing of emulsions and oils, respectively. The results of calculations and experiments at actual specifications of the rotary disintegrator of "Oil Tech Production" company (Table 2) are shown in Fig. 6, on the right.

\subsection{Discussion}

Computational experiments have established that cavitation may occur as a result of elastic deformation, which creates alternating pressure of the liquid in the channel (Fig. 4). It becomes significant when the pressure of the full collapse of the bubble, $p_{\max }$, becomes greater than $p_{0}$ (Fig. 5). It depends on the size of the hole $a$ and the size of the gap between the stator and rotor $\delta$. The maximum number of holes is limited by the size $b$. When the number of holes is maximum, the dependence of $\Delta P=f\left(\frac{a}{\delta}, \max N, \max n\right)$ changes monotonically and shows a maximum (Fig. 6, on the left). It was calculated that $\Delta P$ in the rotary-pulsating disperser РИКА-200 at a pressure in the working volume as used in the disintegrator «Oil Tech Production» company (equal to 2 bar), is less than that at the optimal size $a, \delta$ and $b$ obtained in accordance with the developed theory. Assuming that this difference will be remaining unchanged at all values of $p_{0}$, we can find a range of sizes for $a$ and $\delta$, where $\Delta P$ of rotary disintegrator is always higher than that in РИКА-200:

$$
14 e^{0,6 \ln p_{0}} \delta<a<32 e^{0,7 \ln p_{0}} \delta
$$

Then the minimum of size $b$ in the real range of $p_{0}$ and at the selected level of error $\varepsilon=0,05$ can be expressed as $b$ $>a+1,5 \delta$. In practice, these requirements may be applied to other circumstances, for example, for the maximum 
cross-section of fluid flow through the disintegrator. The mathematical model and study the rotary disintegrator, which has been designed with its use also allowed establishing the fact that such disintegrators satisfy the concept of food sonochemistry in frequency and intensity of cavitation (Fig. 7).

\section{Conclusions}

The use of acoustic and hydrodynamic cavitations in selected applications has been highlighted. It has been suggested that the hydrodynamic cavitation can be an effective alternative for acoustic cavitation when it comes to processing large volumes of processing liquids. The optimization of rotary disintegrators generating strong hydrodynamic cavitation can be achieved on the basis of the theoretical approach discussed in this manuscript. The hydrodynamic cavitation generated in rotary disintegrators can be used in food processing applications where acoustic cavitation has been used. In addition to generating efficient physical forces, hydrodynamic cavitation seems to be more efficient for processing large quantities of food systems (for example, processing of milk in an industrial scale). One of the practical issues of using the rotary disintegrators is the high noise level generated during their operation. Such issues can be easily overcome by constructing a noise proof case around the equipment.

\section{References}

Ashokkumar M., Bhaskaracharya R., Kentish S., Lee J., Palmer M., \& Zisu B. (2010). The ultrasonic processing of dairy products. Dairy Science and Technology, Vol 90, p 147-168.

Ashokkumar M., Sunartio D., Kentish S., Mawson R., Simons L., Vilkhu K., \& Versteeg C. (2008). Modification of food ingredients by ultrasound to improve functionality: A preliminary study on a model system. Innovative Food Science and Emerging Technologies, Vol 9, p 155-160.

Akbarzade K., Hammami A., Harrat A., Zhang D., Allenson S., Jefferson K. Kabir Sh., Jamal A., Marshall A., Rogers R., Mullins O., \& Solbakken T. (2007). Asphaltenes: problems and prospects. Oil and Gas Review. Summer, 28-53 (in Russian).

Balabyshko A., Zimin A., \& Różycki V. (1998). Hydromechanical dispersing. Moscow. Nauka. (in Russian).

Dezhkunov N., Ignatenko P., \& Kotukhov A. (2007). Optimization of the activity of cavitation generated by pulsed ultrasound. Electronic Journal "Technical Acoustics", [Online] Available: http://www.ejta.org, 16 (in Russian).

Duckworth R. B. (Eds.). (1975). Water relations of foods. London: Academic Press.

Flannigan D. \& Suslik K. (2005). The Theory of supercompression of vapor bubbles and nano-scale thermonuclear fusion. Nature, Vol 434, p 52-55.

Floris F. (2004). Nonideal Effects on the Excess Volume from Small to Large Cavities in TIP4P Water. J. Phys. Chem. B, Vol 108, p 16244-16249.

Hint J. (1981). UDA-technology: challenges and perspectives. Tallinn. Valgus (in Russian).

Khavroshkin O., \& Bystrov V. (2007). Sonoluminescence and Sono-Fusion. Applied Physics, Vol 5, p 7-14 (in Russian).

Knapp R., Daily J., \& Hammitt F. (1970). Cavitation. NY: McGraw Book Company.

Klotz A.R., \& Hynynen K. (2010). Simulations of the Devin and Zudin modified Rayleigh-Plesset equations to model bubble dynamics in a tube. Electronic Journal "Technical Acoustics", [Online] Available: http://www.ejta.org, 2010, 11

Krefting D., Mettin R., \& Lauterborn (2004). W. High-speed observation of acoustic cavitation erosion in multibubble systems. Ultrasonics Sonochemistry, Vol 11, P 119-123

Lahey R. T. Jr., Taleyarkhan R. P., \& Nigmatulin R. I. (2007). Sonofusion technology revisted. Nuclear Engineering and Design, Vol 237, p 1571-1585.

Margulis M. (1995). Sonochemistry and Cavitation. London: Gordon \& Breach.

Margulis M. A. (2004). Sonochemistry - a new promising field of high-energy chemistry. Chemistry of High Energies. Vol 38, p 3 (in Russian).

Mawson R., Knoerzer K. (2007). A brief history of the application of ultrasonics in food processing. $19^{\text {th }}$ ICA Congress, Madrid. 
Mettin R., Koch Ph., \& Lauterborn W. (2006). Modeling acoustic cavitation with bubble redistribution. $6^{\text {th }}$ International Symposium on Cavitation, Wageningen

Nigmatulin R. (2005). Nano-scale thermonuclear fusion in imploding vapor bubble. Nuclear Engineering and Design, Vol 235, p 1079-1091.

Nigmatulin R., Akhatov I., Topolnikov A., Bolotnova R., Vakhitova N., Lahey (Jr.) R., \& Taleyarkhan R. (2005). The Theory of supercompression of vapor bubbles and nano-scale thermonuclear fusion, Physics of Fluids, Vol 17, p 107106.

Patent claim WO 2007111524. (2006). International application PCT/RU2006/000140. Biopolymer hydrating method

Promtov M. (2001). Pulsation apparatus of rotary type: theory and practice. Moscow. Mechanical Engineering (in Russian).

Promtov M., Zimin A., \& Monastirsky M. (2001). Model of fluid flow through a single-stage rotary-pulsing apparatus. Industrial Heat Engineering, Vol 23, p 129-133 (in Russian).

Promtov M., \& Monastirsky M. (2000). The dynamics of cavitation bubbles in the high-frequency hydrodynamic emitter of rotary type. International. Conf. "Ultrasonic processes". Severodvinsk: NSTC, p 86-87.

Rozenberg L. (1968). Physics and technology of high-intensity ultrasound. Moskow: Nauka, (in Russian).

Shestakov S. (2001). The basic technology of cavitation disintegration. Moskow: EVA-Press (in Russian).

Shestakov S. (2005). A Mathematical model of hydrodynamic cavitation. XVI session of the Ross. Acoust. Society, Vol 1, p 71-73.

Shestakov S. (2008). Study the possibility of non-parametric amplification multibubble cavitation. Applied Physics, Vol 6, p 18-24.

Shestakov S. (2010). Multibubble acoustic cavitation: A mathematical model and physical similarity. Electronic Journal "Technical Acoustics", [Online] Available: http://www.ejta.org, 2010, 14 (in Russian).

Shestakov S., \& Krasulya O. Research and application experience sonochemical technologies in the food industry. Electronic Journal "Technical Acoustics", [Online] Available: http://www.ejta.org, 2010, 10 (in Russian).

Shlenskaya T., Shestakov S., Krasulya O., Rink R., Smeshek E., Bogush V., \& Artemova Ya. (2011) Ultrasound sonochemistry for hydration of the polar components of environments inverse emulsion in the process of their preparation. XXIV session of Ross. Acoust. Society, Vol 2, p 90-96.

Taleyarkhan R., West C., Cho J.S., Lahey (Jr.) R., Nigmatulin R., \& Block, R. (2002). Evidence for Nuclear Emissions During Acoustic Cavitation. Science, Vol 295, p 1868-1873.

Taleyarkhan R., West C., Cho J., Lahey (Jr.) R., Nigmatulin R., \& Block R. (2004). Additional Evidence of Nuclear Emissions During Acoustic Cavitation. Physical Review, Vol 69, p 036109.

Tikhomirova N., Ashokkumar M., Krasulya O., Shestakov S., \& Bogush V. (2011). Studies in Russia and Australia food sonochemical technologies are becomes jointly. VII International Scientific and practical conference "The latest achievements of science in Europe", Chemistry and Chemical Technology, Sofia, Vol 35, p 64-77. 
Table 1

\begin{tabular}{|c|c|c|c|c|c|c|}
\hline \multirow[b]{2}{*}{ PARAMETER } & \multirow{2}{*}{ 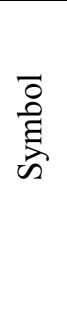 } & \multirow{2}{*}{ 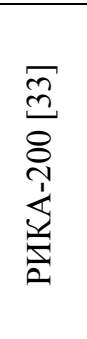 } & \multicolumn{4}{|c|}{$\begin{array}{l}\text { «Oil Tech Production» } \\
\text { disintegrator [31] }\end{array}$} \\
\hline & & & 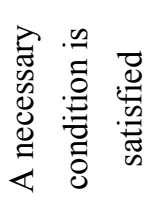 & 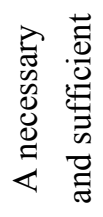 & 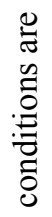 & 胥 \\
\hline Outer radius of the rotor, $m$ & $R$ & 0,1 & 0,1 & 0,1 & & \\
\hline Channel width of stator and rotor, $\mathrm{mm}$ & $a$ & 2,0 & $2,1 / 5,2$ & $2,1 / 5$ & & \\
\hline The number of channels in the rotor & $N$ & 20 & 20 & $147 /$ & & \\
\hline The number of channels in the stator & $n$ & 20 & 20 & $147 /$ & & \\
\hline The pressure in the working volume, $M P a$ & $p_{0}$ & 0,2 & 0,2 & 0,2 & & \\
\hline The angular velocity of rotation of the rotor, $s^{-1}$ & $\omega$ & 340 & 340 & 340 & & \\
\hline The gap between the stator and rotor, $\mathrm{mm}$ & $\delta$ & 0,1 & 0,1 & 0,1 & & \\
\hline A unit capacity of erosion (by cavitation noise), $d B$ & $\Delta P$ & 63,2 & $63,8 / 71,7$ & $81,1 / 8$ & 1,2 & \\
\hline
\end{tabular}

Table 2

\begin{tabular}{|l|c|c|c|}
\hline \multicolumn{1}{|c|}{ PARAMETERS } & & & Note \\
& Value & & \\
\hline Outer radius of the rotor, $m$ & $R$ & 0,072 & \\
Channel width of stator and rotor, $m m$ & $a$ & 2,4 & \\
The number of channels in the rotor & $N$ & 72 & in two rows, total 144 \\
The number of channels in the stator & $n$ & 38 & in two rows, total 76 \\
The pressure in the working volume, $M P a$ & $p_{0}$ & 0,2 & \\
The angular velocity of rotation of the rotor, $s^{-1}$ & $\omega$ & 45 & \\
The gap between the stator and rotor, $\mu m$ & $\delta$ & 26 & \\
\hline
\end{tabular}

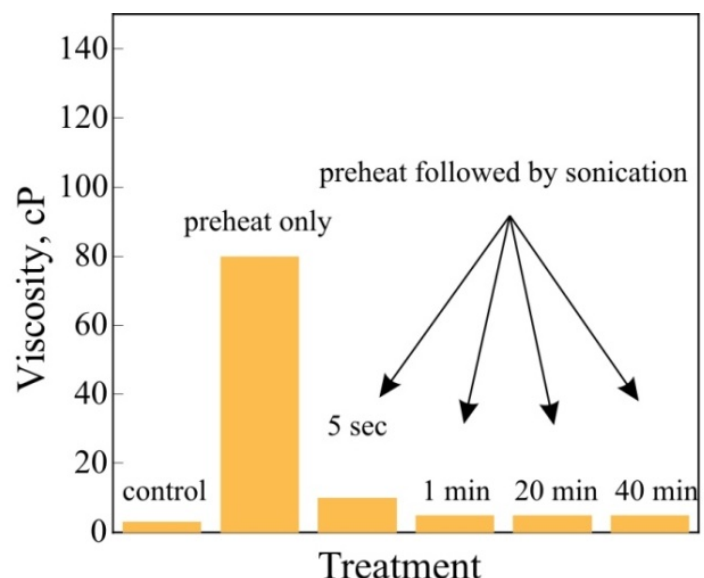

a).

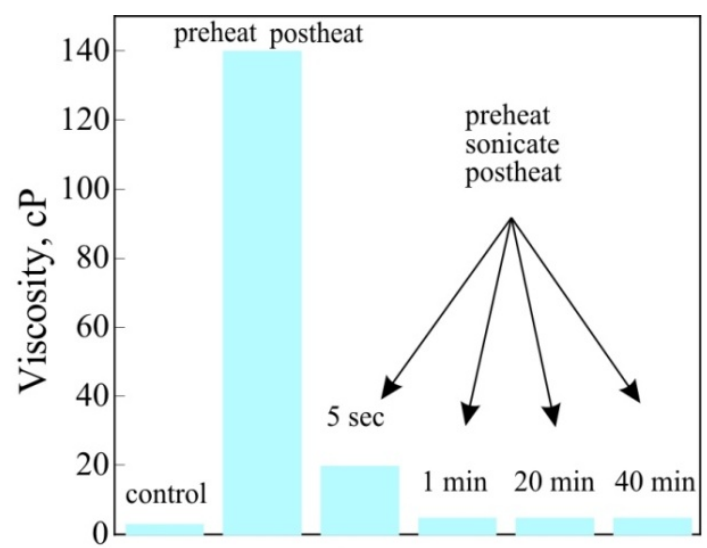

Treatment

b).

Figure 1. Ultrasonic processing of whey proteins provides heat stability in the $9 \%$ solution of WPC

a). Preheated and Sonicated solution. $b$ ). Preheated, Sonicated and Postheated solution 


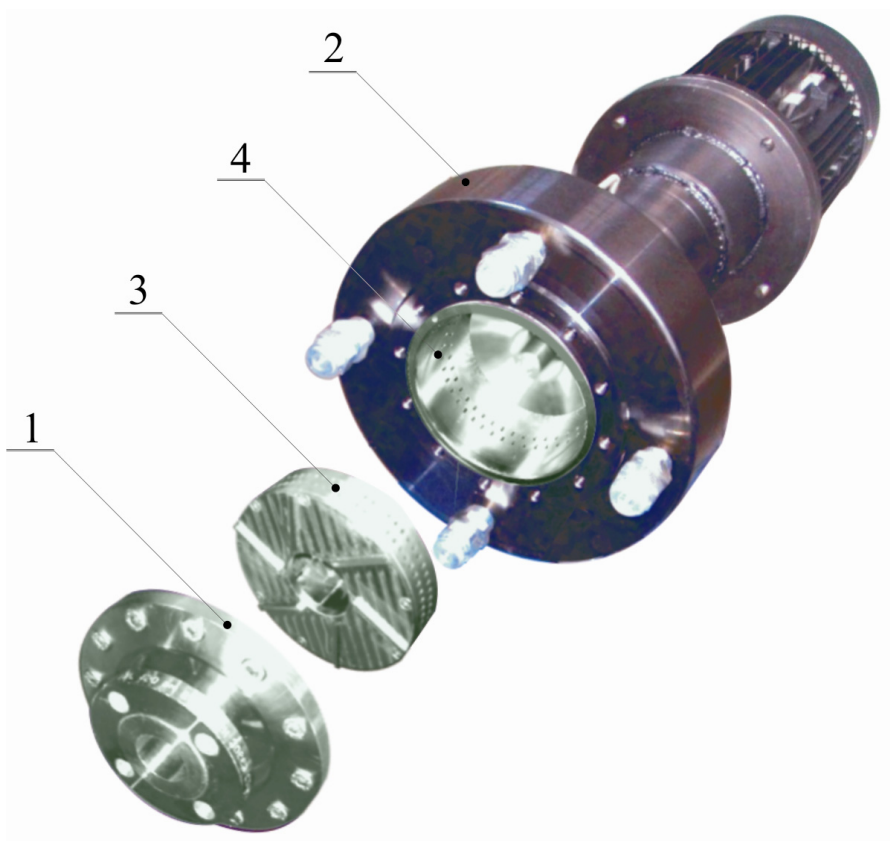

Figure 2. The rotary disintegrator, built in «Oil Tech Production» company with the cover 1, detached from the housing 2 of the working chamber, and rotor 3 , withdrawn from stator 4

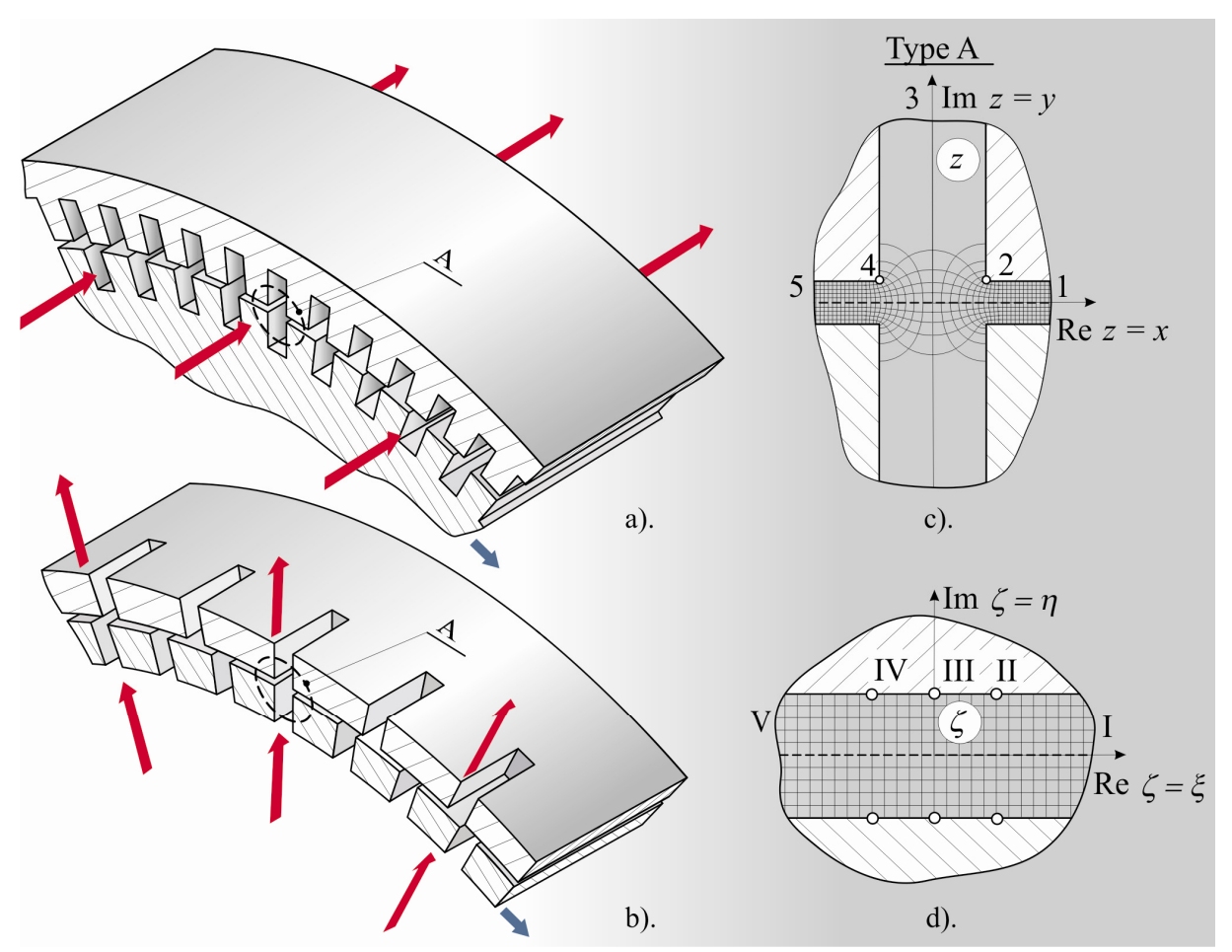

Figure 3. a). Design of rotor and stator of a homogenizer by Swiss company Aquametro AG. $b$ ). The device with the rotor and stator in the form of cylindrical shells, which have rectangular holes. Red arrows indicate the direction of fluid motion, blue - the rotation of the rotor. c). cross section A with the picture of the stress-strain state of the liquid in the gap of the rotor and stator in the absence friction of wall, obtained by means of conformal mapping of strip, shown in $d$ ). $d$ ). the conformal invariant in the form of an infinite strip for cross section A. Arabic and Roman numerals on $c$ ) and $d$ ) indicate corresponding angles 


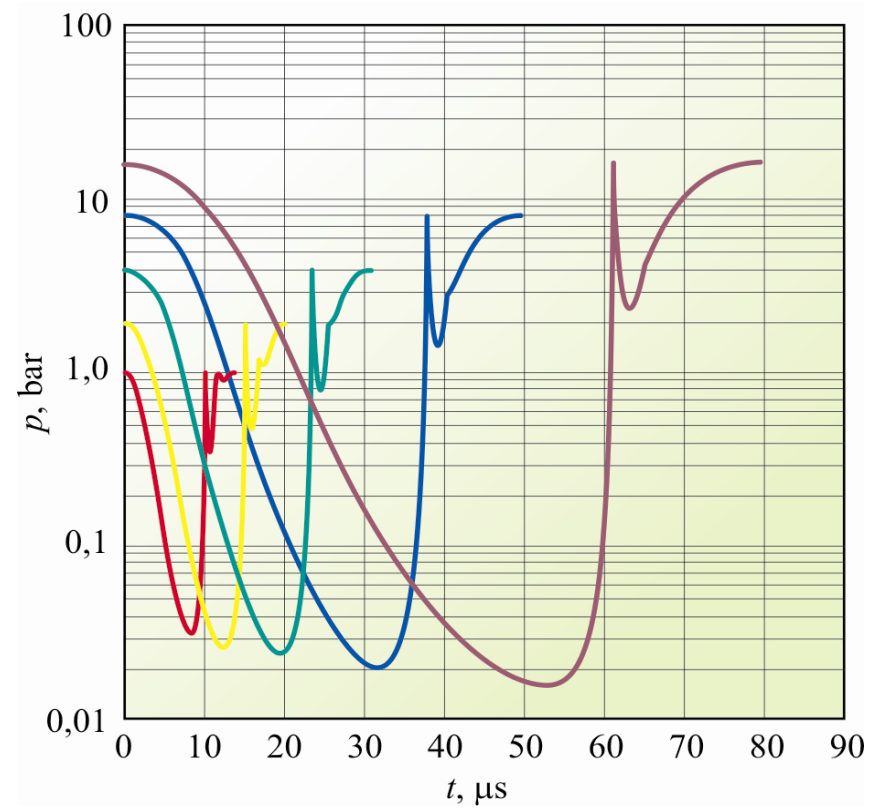

Figure 4. Graphs showing the pressure on the surface of a cavitation bubble that is in the gap between the stator and rotor (Fig. 1, b) with the same ratio sizes $a / \delta=74$ according to the time of opening of the channel $t$ and the pressure in the working volume: -1 bar; -2 bar; -4 bar; -8 bar; -16 bar, respectively

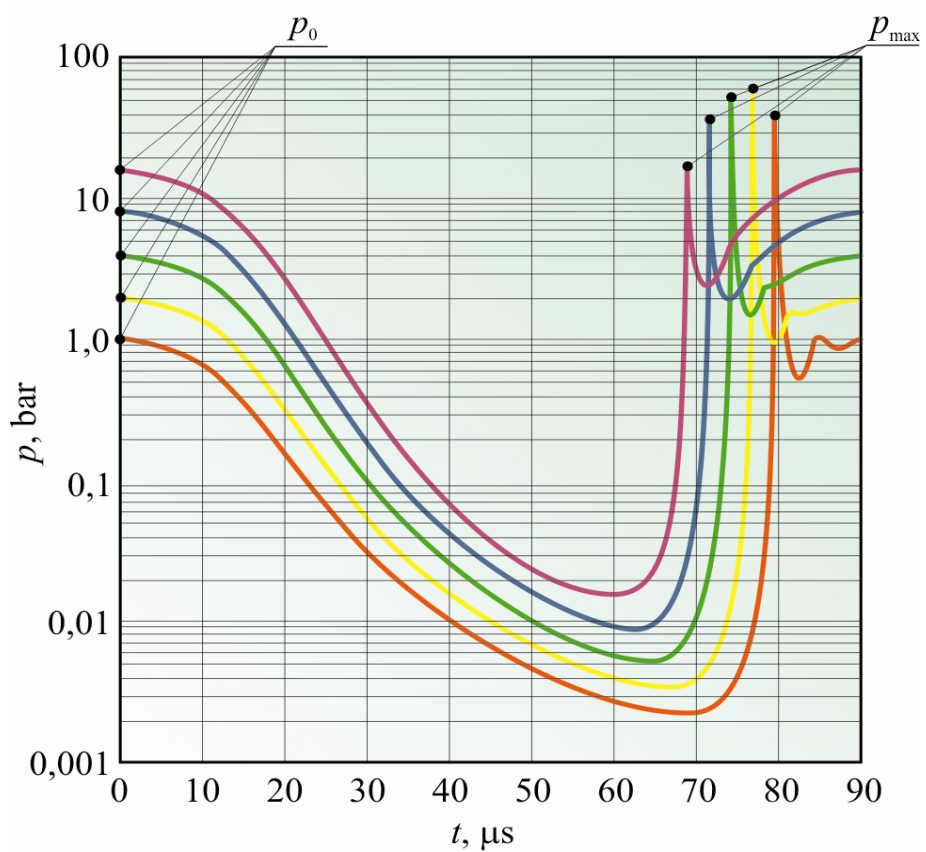

Figure 5. calculated graphs of the pressure on the surface of a cavitation bubble that is in the gap between the stator and rotor (Fig. 1, b) according to the time $t$ of opening of the channel and the pressure in the working volume: -1 bar; -2 bar; -4 bar; -8 bar; -16 bar, respectively, with the ratio sizes $a / \delta$, respectively, $8,13,20,36,74$, when $p_{\max }=p_{0}$ 

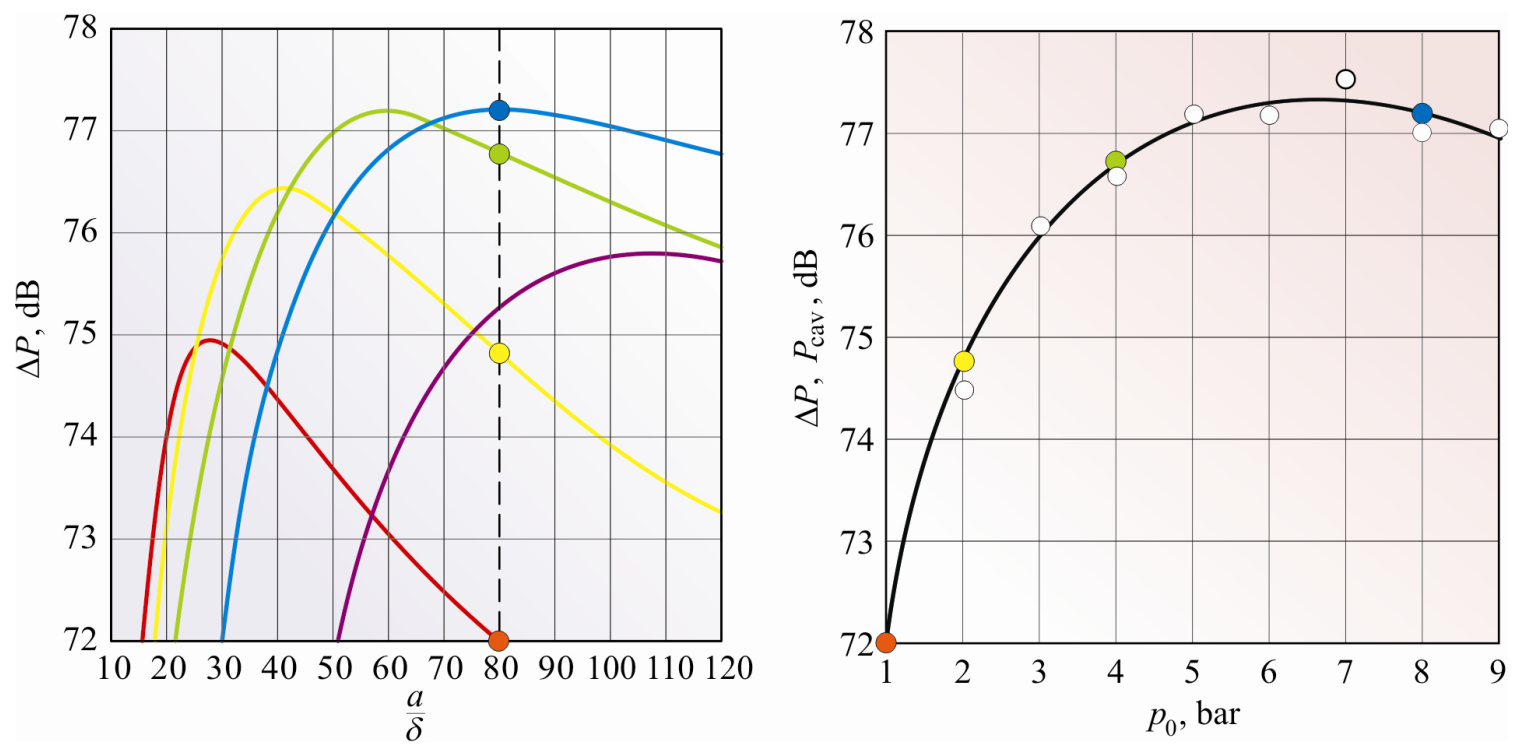

Figure 6. Left - calculated graphs of $\Delta P$ vs $a / \delta$ and pressure in the working volume: -1 bar; -2 bar; -4 bar; -8 bar; -16 bar, respectively. Right - measured $P_{\text {cav }}$ (white circles) and unit capacity of erosion $\Delta P$ (colored dots), dependence on $p_{0},-$ polynomial degrees 3

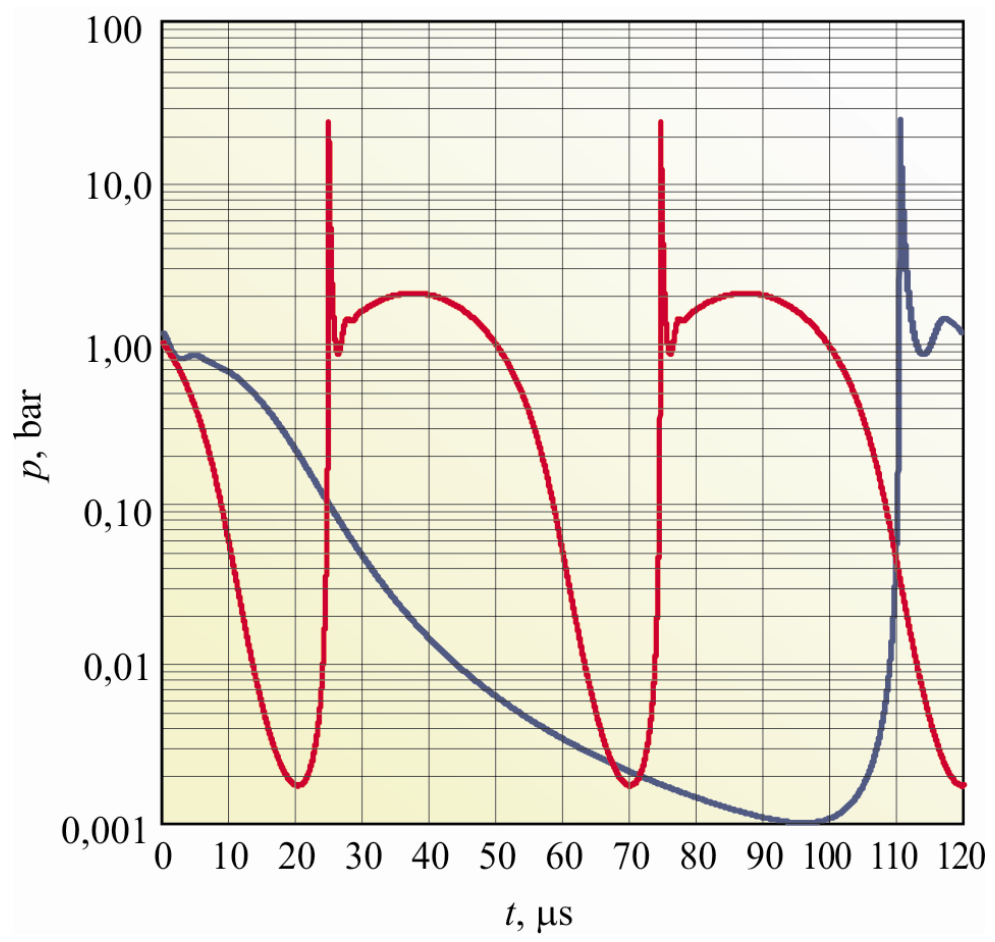

Figure 7. Pressure on the surface of a cavitation bubble as a function of time: - at the antinode of acoustic waves of frequency $20 \mathrm{kHz}$ with an amplitude of sound pressure 1,12 bar; - in the gap between the stator and rotor of the disintegrator при $p_{0}=1,24$ bar 